\title{
NOUVELLE
}

\section{Diversité des mécanismes de transcription des virus géants}

Manon Dassa-Valzer ${ }^{1 *}$, Romain Debiton ${ }^{1 *}$, Margaux Gibert ${ }^{1 *}$, Alexandre Lutz ${ }^{1 *}$, Amel Latifi ${ }^{2}$

\author{
${ }^{1}$ Master 2 Microbiologie Intégrative et Fondamentale, Aix \\ Marseille Université, Marseille, France. \\ ${ }^{2}$ Aix Marseille Université, CNRS, LCB UMR, 7283, IMM, Marseille, \\ France. \\ manon.dassa-valzer@etu.univ-amu.fr \\ romain.debiton@etu.univ-amu.fr \\ margaux.gibert@etu.univ-amu.fr \\ alexandre.lutz@etu.univ-amu.fr \\ latifi@imm.cnrs.fr
}

> Avec son génome de 1,2 Mb et sa capside de $450 \mathrm{~nm}$, Mimivirus, le premier virus géant identifié en 2003 [1], a marqué une rupture dans le monde microbien. Les techniques de filtration utilisées pour l'isolement des virus avaient en effet occulté l'existence des virus géants dont la taille des capsides entraînait leur rétention par les filtres. Depuis la découverte de Mimivirus, de nombreux autres virus géants ont été identifiés. Ils possèdent des capsides allant de $300 \mathrm{~nm}$ à $2 \mu \mathrm{m}$ et des génomes à $A D N$ double brin variant entre $288 \mathrm{~kb}$ et $2,5 \mathrm{Mb}$. Bien qu'ils possèdent parfois un génome plus grand que certaines bactéries, ce sont des parasites obligatoires d'organismes eucaryotes unicellulaires [2]. Leurs hôtes sont retrouvés dans tous les écosystèmes, y compris certains environnements extrêmes comme les cheminées hydrothermales, ou des environnements plus communs comme les sols, le pergélisol, les eaux ou encore le microbiote intestinal. Bien que des virus géants puissent être véhiculés chez l'homme, leur présence ne s'accompagne pas d'une pathogénicité. Étant donné leur grande diversité, notamment au niveau de leurs génomes et de leurs cycles infectieux,

*Ces quatre auteurs ont de façon égale participé à la rédaction de cet article. il est difficile d'avoir une classification universelle. La diversité génomique est illustrée par une forte représentation de gènes ne possédant aucune homologie ni avec les gènes d'autres familles de virus ni avec le monde cellulaire, mais souvent conservés au sein d'une même famille de virus. Ces gènes sont connus sous le terme de gènes orphelins. Il existe différents mécanismes d'entrée et de sortie des virions, qui aboutissent tous au transfert du contenu de la particule virale dans le cytoplasme de l'hôte et à la libération de particules virales dans le milieu. Les différences inter-virus au niveau des cycles infectieux sont liées à la dépendance au noyau cellulaire pour les mécanismes de réplication de I'ADN et de transcription des gènes. Cet article résume les différents mécanismes de transcription connus à ce jour à travers trois exemples majeurs.

\section{Mimivirus, le géant de l'autonomie}

La famille des Mimiviridae est la première famille de virus géants identifiée $[11,12](\rightarrow)$.

Ils sont capables de réaliser leurs cycles infectieux de façon quasiautonome, pour les fonctions nucléaires, dans leur hôte cellulaire. En effet, immédiatement après l'infection, les Mimiviridae initient la transcription de leur génome. Ceci est possible car ils possèdent tous les gènes permettant la synthèse d'une machinerie de transcription autonome (ARN polymérase virale). Ils utiliseront ensuite la machinerie de traduction de I'hôte pour traduire leurs ARN messagers. Ils sont de plus capables d'embarquer cette machinerie dans leurs capsides. Ceci va permettre la transcription des gènes précoces immédiatement après l'infection (Figure 1). Ces derniers gènes présentent un promoteur unique, conservé, différent des promoteurs cellulaires et de ceux utilisés par les gènes tardifs [3]. En plus d'être autonomes pour la transcription, ces virus le sont également pour la maturation de leurs ARNm (voir Encadré «maturation des ARN messagers ») grâce à leur capacité d'intégrer les facteurs de maturation dans leur capside. En 2009, l'analyse des génomes de ces virus a permis de proposer un mécanisme selon lequel un ARN palindromique gouverne l'arrêt de la transcription et la maturation des transcrits (règle de l'épingle à cheveux) [4]. Ce mécanisme, caractérisé en 2015, est assuré par la polyA polymérase virale 


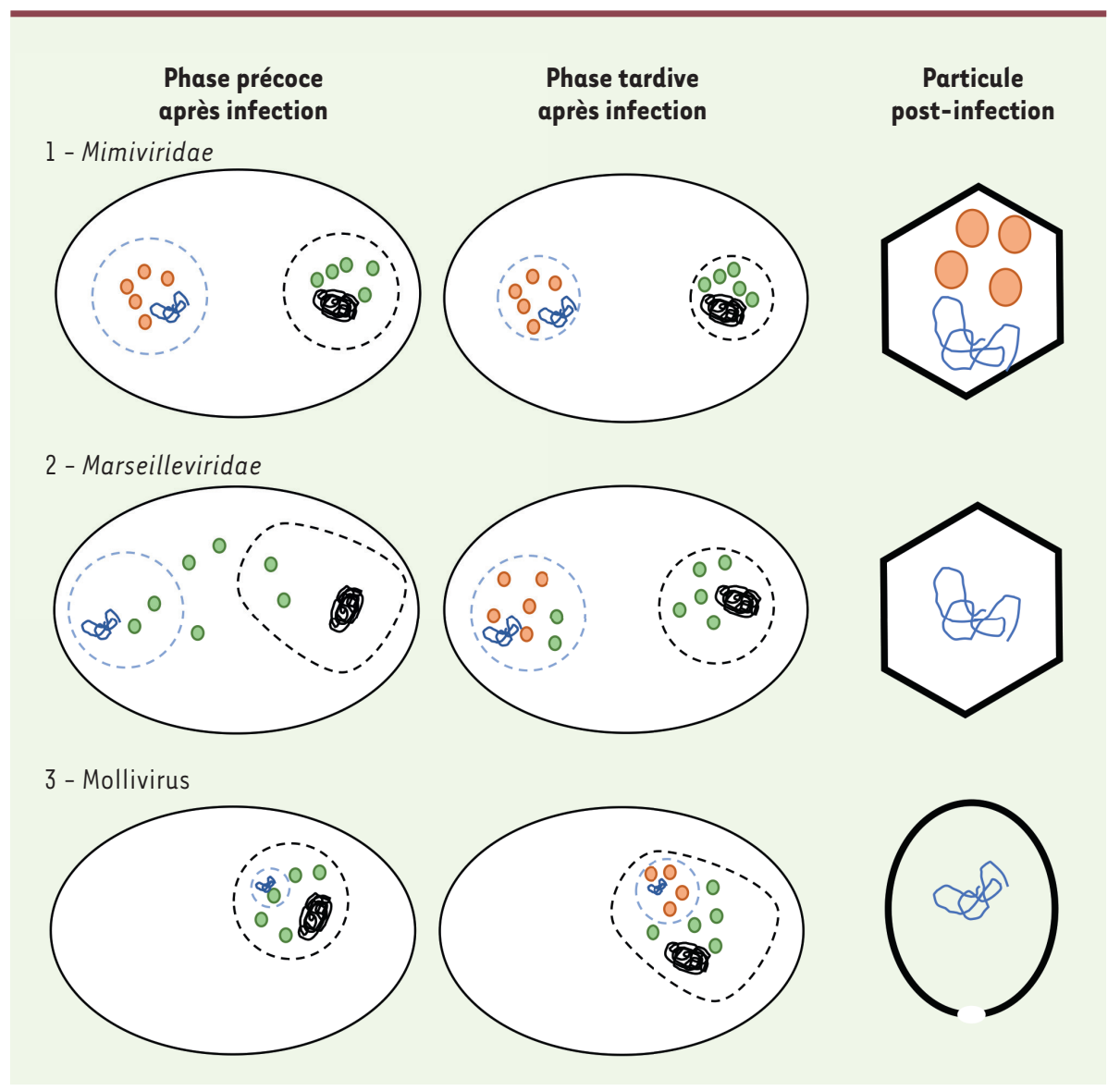

Figure 1. Représentation schématique de différents mécanismes de transcription chez les virus géants. 1. Le virus commence la transcription de ses gènes précoces dans l'usine virale en utilisant sa propre machinerie. Dans la phase tardive, le virus va continuer son cycle infectieux de façon autonome, pour finalement libérer des particules virales contenant son génome ainsi que sa propre machinerie de transcription. 2. Le virus va recruter la machinerie de transcription de l'hôte, en la faisant migrer du noyau vers l'usine virale. Ceci a pour effet de déstructurer le noyau de l'hôte. Par la suite, le virus utilise sa propre machinerie de transcription néosynthétisée pour la transcription des gènes tardifs. Finalement, il libèrera des particules virales contenant son génome, mais sera incapable d'encapsider sa propre machinerie. 3. L'usine virale va se former dans le noyau de l'hôte et détournera la machinerie de transcription de ce dernier. En phase tardive, le virus continue son cycle infectieux dans le noyau mais en utilisant sa propre machinerie néosynthétisée, conduisant à la déstructuration du noyau. La capside virale libérée contient le génome viral. Traits pleins noirs : ADN de l'hôte. Traits pointillés noirs : noyau de l'hôte. Traits pleins bleus : ADN viral. Traits pointillés bleus : usine virale. Ronds verts : machinerie de transcription de l'hôte. Ronds orange : machinerie de transcription virale.

qui présente une structure originale et la capacité unique d'ajouter de longues queues polyA au niveau des structures en épingle à cheveux [5]. Ceci augmente la stabilité des ARNm viraux, retardant leur dégradation, favorisant ainsi la traduction virale par rapport à la traduction cellulaire. Ce sont ces spécificités qui vont différencier les Mimiviridae des autres virus présentés dans cet article.

Mollivirus sibericum, le géant casanier Mollivirus sibericum, virus géant isolé dans le pergélisol sibérien, est un exemple intéressant de virus détournant la transcription de son hôte en installant son usine virale au sein du noyau cellulaire. Une approche de protéomique a permis de décrypter toutes les étapes du cycle de ce virus, ce qui a permis d'avoir une vision globale de cette infection [6]. Cette étude a montré que, bien que codant pour une ARN polymérase, celleci ne se retrouve pas dans la capside. Lors de la phase précoce, la machinerie cellulaire permet la production de l'ARN polymérase virale, qui prend le relais pour le reste du cycle infectieux. La microscopie à fluorescence a également montré le transport de l'ADN viral vers le noyau cellulaire dès le début de l'infection. Toutefois le mécanisme précis du transfert de celui-ci à travers le cytoplasme vers le noyau cellulaire reste encore à élucider. Une fois le matériel viral introduit dans le noyau, ce dernier se déstructure partiellement, permettant ainsi l'accès de l'ADN viral à la polymérase cellulaire (Figure 1). Ce mécanisme est également partagé avec les virus de la famille des Pandoraviridae $[7,8]$, pourtant très éloignés de Molliviridae. Ces virus s'appuient ainsi sur la machinerie de transcription cellulaire en la détournant à leur profit.

Les cycles infectieux des deux familles virales présentés ci-dessus possèdent ainsi des niveaux de dépendance nucléaire opposés, mais il existe d'autres virus ayant un niveau de dépendance intermédiaire.

\section{Marseilleviridae, le géant indécis}

Les Marseilleviridae ont été considérés pendant longtemps comme des virus exclusivement cytoplasmiques parce qu'ils codent une machinerie de transcription complète. Or l'analyse du protéome des virions a révélé l'absence 


\section{Entretien avec Chantal Abergel mené par les auteurs de la Nouvelle}

Chantal Abergel est chercheure en microbiologie avec un tropisme particulier pour la virologie. Suite à son implication dans la découverte et la caractérisation d'un nouveau groupe de virus, les «virus géants » en 2003, elle consacrera le reste de sa carrière à l'étude de cette nouvelle famille émergente. Elle est la co-fondatrice du laboratoire «Information Génomique et Structurale» (IGS-

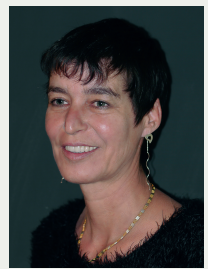

Marseille Luminy) au sein duquel elle exerce en tant que directrice de recherche. Elle a reçu la médaille d'argent du CNRS en 2014 et le prix « La Recherche » en 2015, ainsi que la distinction d'Officier de l'Ordre National du Mérite en 2019.

\section{Quel est votre parcours académique?}

\section{Chantal Abergel}

Parcours universitaire à l'université de Marseille-Luminy jusqu'en Master 1. Après, j'ai quitté pour un moment le cursus car je voulais partir en Amérique du Sud pour faire mon Master 2, mais la personne que je devais rencontrer à Genève pour démarrer ce Master 2 n'est jamais venue... J'ai donc interrompu mon cursus à ce moment. J'ai travaillé au Service de santé des armées en parasitologie en cherchant à acquérir des connaissances dans ce domaine. Cela m'a permis d'intégrer un Master l'année d'après dans un laboratoire qui travaillait sur les calculs pancréatiques. Malheureusement, c'était la première année de cette formation et le responsable avait oublié de demander les bourses de thèses... II a donc fallu que je recherche une thèse. Par chance, une bourse CIFRE co-financée par Matra espace était proposée par un laboratoire qui travaillait sur la cristallisation des protéines. Ayant des connaissances dans ce domaine, j'ai obtenu cette bourse. Le projet de thèse portait sur l'étude des paramètres critiques à la cristallisation des protéines, dont la microgravité. J'ai donc fait une thèse en science des matériaux et j'ai pu faire voler des cristaux dans l'espace! Après ma thèse, je suis partie cinq ans en post-doctorat aux NIH (National Institutes of Health) à Bethesda, aux États-Unis.

Comment a été créé le laboratoire Information Génomique et Structurale? CA : De retour en France, avec Jean-Michel Claverie,nous avons eu l'opportunité en 1995 de créer un laboratoire de bio-informatique et de biologie structurale: I'IGS. Nous nous sommes alors intéressés à des projets qui étaient centrés sur l'analyse et la comparaison des génomes. On a donc démarré un petit programme de génomique structurale qui portait sur la production des protéines et leur caractérisation structurale. Nous avons également eu un financement important en proposant un projet pour rechercher de nouvelles cibles à de nouveaux antibiotiques en comparant les génomes de bactéries pathogènes à ceux des bactéries non pathogènes.

Comment l'IGS s'est-il orienté vers les virus géants?

CA : L'expertise du laboratoire en génomique a fait que de nombreuses personnes nous ont contactés pour faire du séquençage et de l'annotation de génomes, notamment des génomes bactériens. Parmi eux, Didier Raoult, qui était un spécialiste des bactéries parasites, a demandé à collaborer avec le laboratoire sur cette thématique. II s'avère qu'aux alentours des années 2000, un échantillon est arrivé d'Angleterre dans le laboratoire de Didier Raoult dans le but de caractériser l'organisme pathogène infectant les amibes. Notre laboratoire a été impliqué sur l'aspect génomique de ce travail et c'est nous qui avons procédé à l'annotation du génome. Et c'est là que nous avons découvert Mimivirus. Cela a été une fascination immédiate à cause de toutes les surprises que présentaient Mimivirus. On a décidé de ne pas lâcher les virus géants et on a basculé vers un dévelop- pement de nouvelles méthodes qui nous permettaient de les étudier. $\varepsilon t$ à partir de là, nous avons non seulement continué à travailler sur Mimivirus mais nous avons également cherché à isoler d'autres virus géants pour démontrer qu'ils étaient ubiquitaires, qu'on les avait juste occultés à cause d'un processus de filtration utilisé depuis longtemps et imposé par des traditions historiques

Actuellement, qu'est-ce qui est fait dans votre laboratoire?

CA : Nous essayons de faire le plus de choses possibles. C'est-à-dire qu'on utilise les techniques et les méthodologies qui existent à l'heure actuelle pour essayer d'avoir le plus de réponses sur les cycles infectieux de ces virus, sur leur mode de fonctionnement, le rôle des protéines de ces virus et l'interaction qui existe entre ces virus et la cellule, voire même dans des systèmes plus complexes (virus géant, virophage, transpoviron). On utilise toutes les techniques d'imagerie possibles. On essaye également d'implémenter les mutations dans la cellule pour pouvoir réaliser des études fonctionnelles sur les gènes essentiels pour ces virus. Nous faisons de la résolution de structure, en tentant de comprendre la fonction de chacune de ces protéines avec des questions plus vastes qui sont de rétablir les voies métaboliques. Enfin, nous essayons d'établir des liens entre les protéines qui sont codées dans le génome de ces virus. Globalement, nous essayons de répondre, par toutes les techniques possibles et imaginables, à tout un panel de questions biologiques.

Qu'est-ce qui vous intéresse le plus dans l'étude des virus géants? Est-ce que c'est d'établir toute la mécanistique que va avoir un virus pour son cycle infectieux ou est-ce la découverte d'un nouveau virus?

CA : Les deux. Mais plus que ça, il y a une question de fond qui est « Quelle est la place des virus dans l'évolution? Quelle est la place des virus par rapport au monde cellulaire? ». Et ces virus géants nous ont permis de nous introduire et de nous positionner avec ce genre de questions dans le domaine de la virologie. Redéfinir la notion de virus, les replacer dans un contexte évolutif par rapport au monde cellulaire.

Est-ce vous qui avez eu l'idée d'aller chercher les virus géants à tel ou tel endroit, ou ce sont les recherches qui vous orientent sur le prochain site? CA : L'idée du pergélisol, c'est Jean-Michel Claverie qui l'a eue. Un article publié dans PNAS montrait qu'il était possible de réactiver une plante à partir d'un fragment de fruit congelé dans le pergélisol. S'il était possible de réactiver un organisme aussi complexe qu'une plante à fleurs, réactiver un virus devait être également possible. Donc il a pris contact avec les gens qui travaillent là-dessus. Mais avant ça, on avait une logique. Nous avons organisé notre première mission au Chili, dans le Pacifique, car c'est un lieu où il y a beaucoup de nutriments, beaucoup de brassages qui permettent d'avoir un biotope hyper-varié et très riche. Progressivement, on s'est rendu compte qu'en ramassant un peu de terre sous l'arbre situé à l'entrée du laboratoire, il était possible de réactiver des Pandoravirus. Au début, on ne pensait pas qu'ils étaient aussi abondants dans l'environnement. Maintenant, on le sait, ils sont partout et on veut tous les attraper, comme les Pokémon!

Quel est votre virus géant préféré, est-ce qu'il y en a un qui sort un peu du lot?

CA : Chacun sort du lot à sa manière, mais mes préférés sont les Pandoravirus car ils sont tellement différents de tous les virus à ADN ! Il y à $90 \%$ du génome dont on n'a pas la moindre idée de la fonction. Donc je pense que ce sont ceux-là qui peuvent avoir des voies métaboliques originales. On a découvert récemment qu'ils étaient capables de créer leurs propres gènes $a b$ initio. Au niveau évolutif, c'est magistral car cela veut dire que ces gènes n'ont pas d'histoire. II y a encore de la «créativité » qui peut apparaître. 


\section{Maturation des ARN messagers}

Chez les eucaryotes, I'ADN est transcrit en ARN-pré messager qui sera ensuite transformé en ARNm. II existe trois grandes modifications: I'addition de la coiffe à l'extrémité 5', permettant la protection du transcrit contre la dégradation, son exportation vers le cytoplasme, et sa traduction. Elle consiste en la modification du premier nucléotide transcrit, catalysée par la guanylyl transférase et la guanine-7-méthyltransférase. Suite à cela, l'étape d'épissage consiste en l'excision des introns (séquences non codantes de I'ARN primaire) et en l'assemblage bout à bout des exons (séquences codantes). Ces deux étapes se font de manière co-transcriptionnelle. Pour finir, l'addition d'une queue polyadénylée (200 nucléotides environ) à l'extrémité 3 ', est une étape post-transcriptionnelle réalisée par la polyA polymérase, permettant la stabilité de l'ARNm et sa traduction. La maturation de l'ARNm est un processus indispensable à sa traduction [10]. Les virus vont donc aussi devoir effectuer ces modifications afin de pouvoir réaliser la traduction de leurs ARN et donc effectuer leur cycle infectieux.

d'ARN polymérase, ce qui impose l'utilisation de la machinerie cellulaire, au moins pour les gènes précoces [9]. En effet, une étude par microscopie électronique et microscopie à fluorescence a montré, en 2017, que, lors de la phase précoce, le noyau de la cellule hôte se déforme pour permettre la migration de protéines du noyau de la cellule hôte vers l'usine virale. Ce processus se fait sans la libération de I'ADN de l'hôte, éliminant l'hypothèse d'une dégradation totale du noyau. Le modèle proposé pour ces virus est qu'ils seraient capables de recruter la machinerie de transcription de leur hôte, en l'extrayant du noyau et en la faisant migrer vers l'usine virale formée dans le cytoplasme (Figure 1). Ce phénomène leur permettrait d'assurer la transcription des gènes précoce, notamment les gènes codant pour la machinerie de transcription et de maturation des ARNm viraux. La migration des protéines nucléaires et la localisation cytoplasmique de l'usine virale reflètent un mécanisme de dépendance au noyau intermédiaire à ceux vus précédemment.

\section{Synthèse et ouverture sur \\ les mécanismes de transcription chez les virus géants}

Depuis leur découverte, les virus géants n'ont cessé de susciter l'intérêt, notamment à cause de la diversité des environnements où ils sont retrouvés, de leurs tailles, formes, cycles infectieux, etc. Dans cet article, nous avons synthétisé les études récentes réalisées sur les mécanismes de transcription virale et catégorisé au moins trois grands procédés. Les virus les plus autonomes, qui possèdent l'intégralité des protéines nécessaires à la transcription et à la maturation de leur ARNm, illustrés ici par les Mimiviridae dont la transcription est cytoplasmique. Un second système à l'opposé de ce dernier, est utilisé par Mollivirus sibericum et les Pandoraviridae qui, eux, sont nucléaires et détournent la machinerie de transcription de l'hôte en phase précoce. Enfin, les Marseilleviridae utilisent un système intermédiaire, qui requiert la migration de la machinerie de l'hôte du noyau vers l'usine virale. Les données rassemblées jusqu'à ce jour ne permettent pas d'avancer un réel lien évolutif entre tous ces mécanismes, ni d'éliminer l'hypothèse que l'un d'entre eux puisse être la version la plus aboutie. Toutefois, si un parallèle est réalisé avec le schéma évolutif le plus fréquent chez les pathogènes intracellulaires, qui consiste en une réduction génomique, favorisant le détournement des machineries de leur hôte, il serait possible d'imaginer une évolution d'un système de cycle viral cytoplasmique et autonome vers un système nucléaire et dépendant de l'hôte. L'évolution des virus géants est une des questions ouvertes et des plus intéressantes qui restent à élucider en virologie. Ainsi, il se peut que des études plus approfondies des cycles infectieux et des nombreux gènes viraux orphelins permettent de trouver un début de réponse à cette question. $\diamond$ Diversity of transcriptional mechanisms in giant viruses

\section{LIENS D'INTÉRÊT}

Les auteurs déclarent n'avoir aucun lien d'intérêt concernant les données publiées dans cet article.

\section{RÉFÉRENCES}

1. Scola BL. A giant virus in Amoebae. Science 2003; $299: 2033$

2. Brandes N, Linial M. Giant viruses. Big surprises. Viruses $2019 ; 11: 404$

3. Raoult $D$. The 1.2-megabase genome sequence of mimivirus. Science 2004 ; 306 : 1344-50.

4. Byrne D, Grzela R, Lartigue A, et al. The polyadenylation site of mimivirus transcripts obeys a stringent hairpin rule. Genome Res 2009 ; 19 : 1233-42.

5. Priet $\mathrm{S}$, Lartigue A, Debart F, et al. mRNA maturation in giant viruses: variation on a theme. Nucleic Acids Res $2015 ; 43$ : 3776-88.

6. Legendre M, Lartigue A, Bertaux L, et al. In-depth study of Mollivirus sibericum, a new 30,000-y-old giant virus infecting Acanthamoeba. Proc Natl Acad Sci USA 2015 ; 112 : E5327-35.

7. Philippe N, Legendre M, Doutre G, et al. Pandoraviruses: Amoeba viruses with genomes up to $2.5 \mathrm{Mb}$ reaching that of parasitic eukaryotes. Science 2013 ; $341: 281-6$.

8. Pereira Andrade AC dos S, Victor de Miranda Boratto P, Rodrigues RAL, et al. New isolates of pandoraviruses: contribution to the study of replication cycle steps. J Virol 2018 ; 93 : e01942-18/jvi/93/5/JVI.01942-18. atom.

9. Fabre $\varepsilon$, Jeudy S, Santini S, et al. Noumeavirus replication relies on a transient remote control of the host nucleus. Nat Commun 2017 ; 8 : 15087.

10. Bentley DL. Coupling mRNA processing with transcription in time and space. Nat Rev Genet 2014 ; $15: 163-175$.

11. Bekliz M, Levasseur A, La Scola B, Raoult D. MIMIVIRE, un système de défense chez mimivirus qui illustre l'hypothèse de la Reine Rouge. Med Sci (Paris) 2016 ; $32: 818-9$.

12. Claverie JM, Abergel C. Les virus géants. État des connaissances, énigmes, controverses et perspectives. Med Sci (Paris) 2016; 32 : 1087-96.
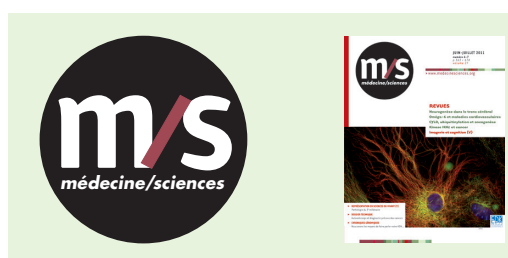

Abonnez-vous

à médecine/sciences

Bulletin d'abonnement page 426 dans ce numéro de $\mathrm{m} / \mathrm{s}$ 